\title{
Deferred Imitation of Action Sequences in Developmental Amnesia
}

\author{
Anna-Lynne R. Adlam, Faraneh Vargha-Khadem', \\ Mortimer Mishkin ${ }^{2}$, and Michelle de Haan ${ }^{1}$
}

\begin{abstract}
The aims of this study were to investigate whether patients with developmental amnesia (DA) associated with bilateral hippocampal volume reduction show an impairment in incidental nonverbal recall of action sequences, and whether the severity of this memory impairment is influenced by the sequence structure (causal vs. arbitrary). Like adult-onset cases of amnesia (McDonough, Mandler, McKee, \& Squire, 1995), patients with DA did not differ significantly from their age-, sex-,
\end{abstract}

\section{INTRODUCTION}

Previous studies of developmental amnesia (DA) have reported impaired episodic memory despite relatively preserved semantic memory (Vargha-Khadem, Salmond, et al., 2003; Vargha-Khadem, Gadian, \& Mishkin, 2001; Gadian et al., 2000; Vargha-Khadem, Gadian, Watkins, et al., 1997). Episodic memory is memory for contextually bound experiences that occur in a particular time and place (e.g., remembering climbing the Eiffel tower last spring), whereas semantic memory is memory for general knowledge (e.g., knowing that Paris is the capital of France), and as such is context-free, including information such as vocabulary and facts about the world and oneself (Tulving, 1972). Although a number of different verbal and nonverbal measures have revealed episodic memory impairment in patients with DA, none of the studies reported so far have examined recall using nonverbal imitation.

In nonverbal imitation, props are used to produce an action or a sequence of actions that the participant is then permitted to imitate, either immediately or after a delay (deferred). The nonverbal reproduction of the event, rather than a verbal description of it, serves as a measure of recall. Typically, two measures of memory are obtained, the number of correct individual actions ("steps") produced and the number of pairs of temporally adjacent steps that are produced in the correct order ("pairs"). Differences between an initial baseline

\footnotetext{
${ }^{1}$ Institute of Child Health, London, UK, ${ }^{2}$ National Institute of Mental Health, Bethesda, MD, USA
}

and IQ-matched controls in spontaneous production of the sequences prior to modeling but recalled fewer target actions and action pairs than the control group after a 24-hour delay, independent of sequence structure. Unlike the patients with adult-onset amnesia, however, the patients with DA showed some memory for both types of sequences after a 24-hour delay. This difference in severity of memory impairment might reflect differences in extent of pathology and/or age at injury.

measure of the spontaneous production of the target actions and their order and performance after exposure to the model are taken as evidence of memory for the event sequence (Bauer, 1997; Mandler \& McDonough, 1995; Meltzoff, 1988).

There is some debate as to what type of memory underlies performance on nonverbal imitation tasks. Some suggest that accurate performance on nonverbal imitation may not necessarily reflect recall from declarative memory. For example, participants might learn a sensorimotor association between an object and an action by observation alone, and thus, presentation of the object might prime the production of the target actions (Werker, 1990). However, according to Mandler (1990), nonverbal imitation tasks require recall (i.e., declarative memory) when the events are entirely novel at the time of the learning session, the participants are not allowed to practice the actions, and the actions are not modeled again after the initial "learning" session (for a detailed discussion, see Bauer, 1997).

Consistent with the latter argument is the finding by McDonough et al. (1995) that patients with adult-onset amnesia are unable to perform an age-appropriate version of the task in either an instructed or uninstructed condition. These investigators tested four groups of participants: frontal lobe patients, amnesic patients, "experienced" (with demonstration) healthy controls, and "inexperienced" (without demonstration) healthy controls, on both instructed and uninstructed nonverbal deferred imitation. Using word-list recall as the supposed true task, the sequences were presented as a set of incidental events, making their presentation 
similar to that described in the child literature. Participants were given the props to manipulate (baseline); then, for all the participants except the "inexperienced" controls, the experimenter demonstrated the target actions. After a 24-hour delay, the participants were tested for recall. In the uninstructed recall condition, the props were placed in front of the participant for 1 minute; in the instructed condition, the participants were asked to produce the actions in the same order as the one demonstrated by the experimenter. The frontal lobe patients and the "experienced" controls produced significantly more target actions and target action pairs in both instruction conditions than in baseline and significantly more than the patients with amnesia and "inexperienced" controls.

In summary, the patients with amnesia failed to perform the sequences they had seen in either the instructed or uninstructed condition. This suggests that the uninstructed condition did not automatically or unconsciously prime them to perform the sequences. Furthermore, the finding that the frontal lobe patients were able to recall the actions and their order suggests that nonverbal imitation is not a task of problem-solving, a function thought to be impaired after frontal lobe injury (e.g., Colvin, Dunbar, \& Grafman, 2001; Goel \& Grafman, 1995). This study is the only study to date to test deferred imitation in patients with amnesia and therefore warrants attempts at replication.

Memory for event sequences is influenced by a number of factors, including the structure of the event. The events to be remembered are made up of sequences of actions that are ordered according to their temporal and causal connections. When one action in an event must be carried out prior to another in order to achieve the desired end-state or goal, the pair of actions is said to be causally ordered. For example, if the desired endstate was transfer of water from one cup to another with a straw, logically the straw must be placed in the cup containing water, a finger must be placed over the top of the straw, and the straw must then be released in the empty cup. Alternative temporal orders of the same actions would not achieve the goal. In contrast, action sequences that are not temporally constrained to reach a goal can be arbitrarily ordered. For example, to balance coins on a ruler resting on a block, the coins can be placed on each side of the ruler before or after the ruler is balanced on a block. In this example, the actions can be performed in either order without preventing the achievement of the final end-state.

Several studies have found that children and adults have superior ordered recall of events that are characterized by causal actions compared with events that lack such relations (e.g., Barr \& Hayne, 1996; McDonough et al., 1995; Bauer \& Mandler, 1992) and that this effect is maintained over a delay (e.g., Mandler \& McDonough, 1995; McDonough et al., 1995; Bauer \& Hertsgaard, 1993). This effect is obtained even when equivalent numbers of individual target actions are produced in both conditions (Bauer, 1996), indicating that the differences in ordered recall are not an artifact of differential opportunities for ordering. Moreover, the performance at baseline (i.e., without exposure to the target actions) does not typically differ between these sequence structures, suggesting that the superior ordered recall of causal sequences is not due to their greater transparency (e.g., Bauer, Hertsgaard, \& Wewerka, 1995; Bauer, 1992, 1996).

It seems, therefore, that the more causal the structure in a sequence, the better the recall of temporal order of the component actions. This is somewhat similar to the superior recall of category-ordered word-lists compared to unordered word-lists often found in healthy adults (e.g., Channon \& Daum, 2000; Channon, Daum, \& Polkey, 1989). Like category-ordered word-lists, the superior recall of causally ordered sequences may be due to their increased meaningfulness compared to arbitrarily ordered sequences. In this way, recall of the order of causally ordered sequences might be considered more "semantic" compared to arbitrarily ordered sequences. Thus, although production of the sequences should be independent of sequence structure at baseline (e.g., Bauer, Hertsgaard, Wewerka, et al., 1995; Bauer, 1992, 1996), patients with DA, having relatively preserved semantic memory, might be less impaired relative to controls at recall of the causally ordered sequences compared to the arbitrarily ordered sequences (i.e., a Group $\times$ Assessment Condition $\times$ Sequence Structure interaction).

The aims of this study were twofold. First, to investigate whether, like patients with adult-onset amnesia (McDonough et al., 1995), memory impairments would be observed in patients with DA using an unalerted memory task. Second, to test whether the severity of the memory impairment would be influenced by the structure of the to-be-remembered material. To this end, the performance of 12 patients with DA was compared to the performance of 12 healthy age-, sex-, and IQmatched controls on the deferred imitation sequences reported by McDonough et al. (1995).

\section{RESULTS}

\section{Actions}

The number of correct target actions performed by each participant during each phase of the experiment was submitted to a $2 \times 2 \times 2$ mixed-design analysis of variance. The between-subjects factor was group (DA, control). The within-subjects factors were assessment condition (baseline, delay) and sequence structure (causal, arbitrary). The mean $( \pm S E M)$ number of arbitrary and causal target actions correctly recalled by each group at baseline and after a 24-hour delay is shown in Figure 1. The analysis revealed that the control group 
performed better than the DA group [main effect of group, $F(1,22)=25.60, p<.05]$, delayed performance was better than baseline performance [main effect of condition, $F(1,22)=212.95, p<.05$ ], and more causal actions were produced than arbitrary actions [main effect of structure, $F(1,22)=8.5, p<.05]$. These main effects were qualified by two significant two-way interactions: Group $\times$ Condition $[F(1,22)=6.70, p<.05]$ and Condition $\times$ Structure $[F(1,22)=8.06, p<.05]$. The predicted Group $\times$ Condition $\times$ Structure interaction was not significant, $p>.05$.

Follow-up analysis of the Group $\times$ Condition interaction revealed that, consistent with the findings reported by McDonough et al. (1995), the groups did not differ significantly on baseline performance $p>.01,{ }^{1,2}$ but the control group recalled more target actions than the DA group after a delay, $t(22)=6.06, p<.01$. Further followup analysis revealed that both groups produced more target actions at delayed recall than at baseline [control, $t(11)=12.42, p<.01 ;$ DA, $t(11)=8.31, p<.01]$, indicating memory for the demonstrated actions. Together these findings suggest that the memory effect was larger in the control group, although an analysis of the difference in the magnitude of the memory effect (performance after delay minus performance at baseline) between the groups just failed to reach statistical significance when corrected for multiple comparisons, $t(22)=2.59, p=.017$.

Follow-up analysis of the Condition $\times$ Structure interaction revealed that more causal than arbitrary actions were produced at baseline, $t(23)=4.10, p<.025,^{3}$ but unexpectedly, not after a delay, $p>.025$.

\section{Action Pairs}

The mean $( \pm S E M)$ number of arbitrary and causal target action pairs correctly recalled by each group at baseline

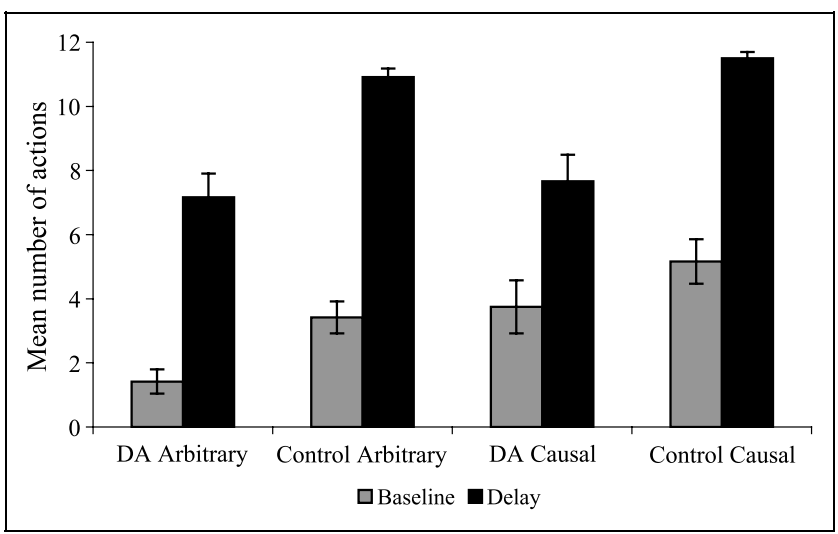

Figure 1. The mean number of target arbitrary and causal actions correctly recalled by each group at baseline and after a 24-hour delay (maximum $=12)$. Standard errors are shown for each score. DA = developmental amnesia. and after a 24-hour delay is shown in Figure 2. A 2 (group) $\times 2$ (condition) $\times 2$ (structure) mixed-design analysis of variance revealed that, again, the control group performed better than the DA group [main effect of group, $F(1,22)=53.14, p<.05]$, delayed performance was better than baseline performance [main effect of condition, $F(1,22)=219.83, p<.05$ ], and more causal actions were produced than arbitrary actions [main effect of structure, $F(1,22)=25.88, p<.05$ ]. There was also a significant Group $\times$ Condition interaction $[F(1,22)=52.49, p<.05]$ but not a Condition $\times$ Structure interaction $[F(1,22)=3.38, p>.05]$. Again, the predicted Group $\times$ Condition $\times$ Structure interaction was not significant, $p>.05$.

Follow-up analysis of the Group $\times$ Condition interaction revealed that, consistent with the findings reported by McDonough et al. (1995), the groups did not differ significantly on baseline performance, $p>$ $.01,{ }^{1}$ but the control group recalled more target action pairs than the DA group after a delay, $t(22)=8.69$, $p<.01$. Further follow-up analysis revealed that both groups produced more target action pairs after a delay than at baseline [control, $t(11)=16.63, p<.01 ;$ DA, $t(11)=5.07, p<.01]$, indicating memory for the action pairs. Together, these results suggest that the memory effect was larger in the control group, a finding confirmed by an analysis showing a significant difference in the magnitude of the memory effect between the groups, $t(22)=7.25, p<.01$.

\section{Task Awareness}

The deferred imitation task was designed to be a test of unalerted recall in that participants were not told that they would be required to recall the sequences after demonstration. Nevertheless, each participant was asked if he/she was aware of the purpose of the task, and if so when that awareness occurred to him or her (see Methods). None of the control group participants

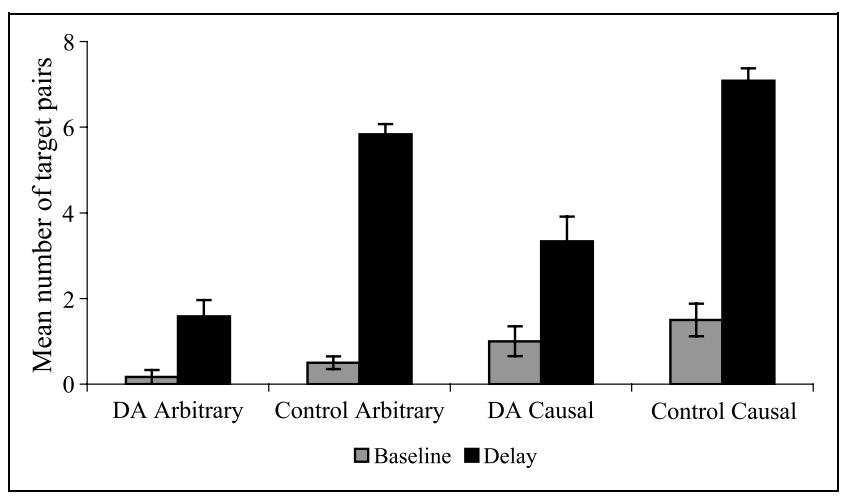

Figure 2. The mean number of arbitrary and causal target pairs correctly recalled by each group at baseline and after a 24-hour delay (maximum $=8)$. Standard errors are shown for each score. DA = developmental amnesia. 
indicated an awareness of the purpose of the task and only one patient (DA1) indicated some awareness of the study aim during the first day (i.e., Phases 1 and 2). This awareness did not affect subsequent recall of the sequences in that overall the patient group was impaired relative to controls even when the scores obtained by Patient DA1 were removed from the analysis.

\section{DISCUSSION}

The aims of this study were to investigate whether patients with DA showed memory impairments in an unalerted memory task involving imitation of action sequences, and to test whether the severity of the memory impairment would be influenced by the structure of the to-be-remembered material. The results indicate that relative to controls, patients with DA were impaired at delayed recall of both target actions and their temporal order but the severity of their memory impairment was not affected by event structure. These impairments appear to be due to memory difficulties rather than general difficulties with the task as the groups did not differ on their baseline performance. Despite their impairment, however, the patients with DA demonstrated some memory for the target actions and their temporal order even after the 24-hour delay.

The findings reported here replicate the findings reported by McDonough et al. (1995), in that the patients with DA were impaired relative to controls at delayed recall of actions and action pairs but not at baseline. This impairment in delayed nonverbal recall is consistent with the previous findings in patients with DA (Vargha-Khadem, Salmond, et al., 2003; Gadian et al., 2000; Vargha-Khadem, Gadian, Watkins, et al., 1997) and the general neuropsychological profile reported here (see Methods). However, the DA group did produce more target actions and action pairs at delayed recall compared to baseline. Although this memory effect was smaller than that in controls (significantly for pairs but only marginally so for target actions), it does indicate that patients with DA were able to recall some information even after a 24-hour delay. The only hint that the patients with adult-onset amnesia reported by McDonough et al. showed any memory for the event sequences was a significant main effect of assessment (baseline vs. uninstructed recall vs. instructed recall) with the mean number of actions increasing in the expected direction. However, post hoc follow-up comparisons of the two memory conditions versus baseline showed no significant effects. Therefore, patients with DA appear to show better memory at delay compared to that shown by the adult-onset amnesia cases reported by McDonough et al. This is not likely to be due to differences in tasks between the studies as the sequences used were identical, as was the method of administration except for the lack of the uninstructed recall phase.
The difference between patients with DA and those with adult-onset amnesia could be due to the differences in extent of injury between these two patient groups. The patients with DA have, within the MTL, selective hippocampal pathology, whereas the patients reported by McDonough et al. are of mixed etiology with some patients having Korsakoff amnesia, a disorder associated with diencephalic and possibly frontal lobe damage. Alternatively, or additionally, the less severe memory deficit in the DA group might be a consequence of their young age at injury, in that patients with DA may have a less severe amnesia due to the functional reorganizational capacity of the immature brain (e.g., Nelson, 2000; but see Vargha-Khadem, Salmond, et al., 2003).

The effects of event structure were generally consistent with the idea that causal structure facilitates memory of the order of an event. Specifically, although the structure variable did not affect the number of target actions produced after a delay, it did affect the number of postdelay action pairs. The finding that more causal target action pairs but not individual target actions were recalled after a delay suggests that the correct recall of the temporal order of causal sequences is not merely due to an increase in the production of causal compared to arbitrary actions. This provides strong evidence that memory for the order of causal sequences is better than memory for the order of arbitrary sequences and is consistent with other studies of nonverbal imitation (Bauer, 1996; Bauer, Hertsgaard, Wewerka, et al., 1995; Mandler \& McDonough, 1995; Bauer \& Hertsgaard, 1993; Bauer, 1992), and category-ordered versus unordered word-list recall (Channon \& Daum, 2000; Channon, Daum, Polkey, et al., 1989). However, the present findings provide no evidence to support the prediction that the DA group would be less impaired relative to controls at recall of the causally ordered sequences compared to arbitrarily ordered sequences. It seems, therefore, that despite the causal sequences potentially being more meaningful than the arbitrary sequences, recall was still largely dependent on episodic memory. The sequences were presented once for study and involved performing unusual actions, such as hanging a clip over a magnet attached to the bottom of a cup. The novelty of such sequences may make them more dependent on episodic memory, and perhaps therefore more dependent on the hippocampus, despite the meaningfulness in the temporal order of the actions (e.g., Strange, Fletcher, Henson, Friston, \& Dolan, 1999). Furthermore, it is possible that the event structure did not affect the performance of patients with DA due to the short sequence length (3 steps) and therefore low task demands. That is, previous studies supporting the advantage of causal relations on recall have tested much younger children/infants (e.g., Mandler \& McDonough, 1995; Bauer \& Hertsgaard, 1993), whereas word-list recall tasks have tested adults using much longer list lengths (Channon \& Daum, 2000; 
Channon, Daum, Polkey, et al., 1989). Additionally, the controls performed close to ceiling on delayed recall of both causal and arbitrary target steps. It is possible that this underestimated their performance, in which case the DA group might have been more impaired at arbitrary sequence recall if the controls had been given the opportunity to demonstrate a higher level of performance. It is therefore possible that, relative to novel sequences, longer event sequences designed more specifically to tap into semantic memory, namely, familiar scripted sequences, might show the predicted effect of a lesser impairment in patients with DA (e.g., Adlam, Gadian, Vargha-Khadem, \& de Haan, 2004).

An unpredicted finding related to event structure was that more causal than arbitrary actions and action pairs were produced at baseline. Although this finding was unexpected on the basis of many previous studies (e.g., Bauer, Hertsgaard, Wewerka, et al., 1995; Bauer, 1992, 1996), it is consistent with the findings of McDonough et al. (1995). As the same sequences were used in the two studies, this result likely reflects the properties of the props involved in these particular sequences. Importantly, both groups showed a similar pattern of baseline performance (i.e., both groups showed better production of causal than arbitrary sequences), and thus, the absence of the predicted Group $\times$ Structure interaction for delayed recall cannot be attributed to baseline differences between the groups.

\section{Conclusions}

We have reported the performance of patients with DA on a task of nonverbal deferred imitation, a measure of incidental delayed nonverbal recall. The findings replicated an earlier study in adult-onset amnesia cases (McDonough et al., 1995), in that delayed memory for the nonverbal sequences was impaired in the DA group relative to controls independent of sequence structure. However, unlike the patients with adultonset amnesia, patients with DA showed some evidence for memory of the sequences even after a 24-hour delay. Whether this superior residual memory in patients with DA relative to patients with adult-onset amnesia is due to differences in extent of pathology and/or age at injury remains to be tested. Moreover, the nature of the residual memory in DA, that is, whether this is supported by episodic or semantic memory, needs to be further examined using sequences designed more specifically to tap into each of these types of memory separately (Adlam et al., 2004).

\section{METHODS}

The method we used was the one described by McDonough et al. (1995) except that, to reduce testing time, we excluded their uninstructed condition, which had given results that did not differ significantly from those of the instructed condition.

\section{Participants}

Twelve patients with DA participated in this study. Details of the patients are presented in Table 1. Each patient had reduced hippocampal volumes bilaterally as noted radiologically and confirmed using volumetrics $[F(1,17)=65.89, p<.05]$ (see Table 1$)$. Twelve healthy normal individuals matched to the patients on age $(p>$ $.05)$, sex $(p>.05)$, and IQ $(p>.05)$, served as controls (see Table 2).

The patients were impaired relative to controls on: the Rivermead Behavioral Memory Test [RBMT, Wilson, Cockburn, \& Baddeley, 1985: $t(22)=11.03, p<.05$ ]; delayed story recall [WMS-adapted, Vargha-Khadem, Isaacs, van der Werf, Robb, \& Wilson, 1992: $F(1,22)=$ 66.16, $p<.05$ ]; delayed design recall [WMS-adapted, Vargha-Khadem, Isaacs, et al., 1992: $F(1,22)=58.77, p<$ $.05]$; delayed recall of the Rey-Osterith complex figure [Rey, 1964: $t(22)=6.58, p<.05]$; and delayed word-list recall [CAVLT-2, Talley, 1993: $t(22)=7.95, p<.05$ ]. However, the groups did not differ significantly on measures of vocabulary (WISC-III/WAIS-III, Wechsler, 1992, 1997: $p>.05$ ), comprehension (WISC-III/WAIS-III, Wechsler, 1992, 1997: $p>.05)$, digit span forward (WISC-III/WAIS-III, Wechsler, 1992, 1997: $p>.05$ ), digit span backward (WISC-III/WAIS-III, Wechsler, 1992, 1997: $p>.05$ ), basic reading (WORD, Rust, Golombok, \& Trickey, 1993: $p>.05$ ), and spelling (WORD, Rust et al., 1993: $p>.05)$.

\section{Stimulus Materials}

There were 8 novel three-step action sequences (as described by McDonough et al., 1995): 4 with causal relations and 4 with arbitrary relations (for a list of objects and action sequences, see Appendix).

\section{Procedure}

For each sequence, the objects were given to the participants for an initial baseline period of 1 minute. Once all of the sequences had been presented for baseline, the experimenter modeled each sequence once without narration. Recall of all eight sequences was tested 24 hours after modeling. The presentation of the tasks was counterbalanced across participants at baseline, and the props were presented for delayed recall in the same order as at baseline. To make the task an incidental memory test, as it is typically given to nonverbal infants and as done in the study reported by McDonough et al. (1995), the sequences were embedded as filler tasks in tests of learning and remembering words (results not reported here). The study was conducted in 
Table 1. Details of Patients with DA

\begin{tabular}{|c|c|c|c|c|c|c|}
\hline \multirow[t]{2}{*}{ Case } & \multirow[t]{2}{*}{ Sex } & \multirow[t]{2}{*}{$\begin{array}{l}\text { Age at Injury } \\
\text { (year:month) }\end{array}$} & \multirow[t]{2}{*}{$\begin{array}{l}\text { Age at Test } \\
\text { (year:month) }\end{array}$} & \multirow[t]{2}{*}{ Etiology } & \multicolumn{2}{|c|}{$\begin{array}{c}\text { Percentage of } \\
\text { Hippocampal Volume Reduction }\end{array}$} \\
\hline & & & & & Left & Right \\
\hline DA1 & M & Perinatal & 19:11 & Birth asphyxia & 56.6 & 40.7 \\
\hline DA2 & $\mathrm{F}$ & Perinatal & $18: 00$ & Birth asphyxia & - & - \\
\hline DA3 & M & Perinatal & $16: 00$ & Birth asphyxia & 34.2 & 32.3 \\
\hline DA4 & M & Perinatal & $14: 01$ & Birth asphyxia & - & - \\
\hline DA5 & $\mathrm{F}$ & Perinatal & $10: 06$ & Birth asphyxia & - & - \\
\hline DA6 & $\mathrm{F}$ & 2 days & $14: 07$ & Hypoxia-ischemia & 48.1 & 43.4 \\
\hline DA7 & M & 11 weeks & $15: 09$ & Hypoxia-ischemia & 34.8 & 33.9 \\
\hline DA8 & $\mathrm{F}$ & 0 to $2: 06$ & $15: 02$ & Unconfirmed seizures & 23.8 & 27.1 \\
\hline DA9 & M & 0 to $4: 06$ & $13: 09$ & Unconfirmed seizures & 21.2 & 30.7 \\
\hline DA10 & $\mathrm{F}$ & 9:01 & $26: 03$ & Hypoxia-ischemia & 34.2 & 37.5 \\
\hline DA11 & $\mathrm{F}$ & $12: 05$ & $19: 07$ & Hypoxia-ischemia & 51.3 & 54.1 \\
\hline DA12 & M & $15: 05$ & $17: 11$ & Hypoglycemia & 53.5 & 42.7 \\
\hline
\end{tabular}

The percentage of hippocampal volume reduction is relative to the control group and is corrected for intracranial volume. The dashed line (-) indicates missing data.

three phases, which were videotaped for later scoring. Phases 1 and 2 were administered on Day 1, Phase 3 was administered 24 hours after Phases 1 and 2. A summary of sessions is shown in Table 3 .

In Phase 1, the experimenter (E) administered the word-list. Then during the imposed word-list delay E told the participant: "Now I am going to have you do some things to keep you from thinking of those words. I will give you different sets of objects, which I want you to handle, manipulate, or do whatever you like with them. It is important that you pay close attention to the objects, so please do not talk while you are handling them." Then E brought out, one at a time, each of the object sets. The participants were given 1 minute to manipulate each object set (baseline condition). Phase 2 followed immediately. E said, "Now I want you to observe some objects while I manipulate them. Please pay close attention, do not talk, and try not to think of the words that I read to you earlier." E then modeled the target actions for each of the eight sets of objects.
After the eighth set of objects was removed, the participant was asked to recall and recognize as many of the words on the list as could be remembered. After the recognition test, $\mathrm{E}$ read the word-list one more time telling the participant that recall of the words would be tested again the next day.

On the next day, Phase 3 began when the participants were asked to repeat the words that they had been read the day before. E then read the words again, instructing the participants to remember them. Then the object sets were placed in front of the participant, one at a time, in the same order as previously presented and E said, "I want you to show me as accurately as you can remember the actions I performed yesterday and the order in which I performed them." After a participant was clearly finished with an object set, it was removed and the next set was presented, and so on, until the participant had an opportunity to manipulate all eight sets.

After Phase 3, participants were questioned to assess their understanding of the purpose of the study.

Table 2. The Mean IQ and Age at Test

\begin{tabular}{lccccr}
\hline & \multicolumn{1}{c}{ Sex } & $\begin{array}{c}\text { Mean Age at Test } \\
\text { (year:month) }\end{array}$ & $\begin{array}{c}\text { Full Scale IQ } \\
\text { Standard Score }\end{array}$ & $\begin{array}{c}\text { Verbal IQ } \\
\text { Standard Score }\end{array}$ & $\begin{array}{c}\text { Performance IQ } \\
\text { Standard Score }\end{array}$ \\
\hline DA & 6 boys, 6 girls & $16: 09(10: 06-26: 03)$ & $90.0(75-114)$ & $91.7(80-108)$ & $90.3(70-121)$ \\
Control & 6 boys, 6 girls & $16: 09(11: 02-26: 06)$ & $97.5(80-114)$ & $95.3(82-113)$ & $101.4(75-128)$ \\
\hline
\end{tabular}

The range is shown for each score. $\mathrm{DA}=$ developmental amnesia. 
Table 3. Example of Phases 1, 2, and 3

Day 1 (a.m.)

Phase 1 Presentation of word-list A

Baseline Set A (eight 3-step novel sequences)

Phase 2 Model Set A

Delayed recall and recognition of word-list A; presentation of word-list A

Day 2 (a.m.)

Phase 3 24-hour delayed recall of word-list A; presentation of word-list A

Delayed recall of Set A

Recall of word-list A

Questions about purpose of task

E asked, "What do you think is the purpose of this experiment? At any point in the experiment did you expect to be asked to demonstrate the actions I performed for you yesterday?" Participants who expressed any understanding of the purpose of the experiment were asked when it occurred to them. These questions were asked in order to determine whether the participant was aware of the purpose of the task (i.e., delayed recall of the action sequences) during the first day of testing, as this awareness may have influenced recall on the second day.

\section{Scoring}

For each sequence, the total number of different target steps produced was calculated, as was the number of pairs of actions produced in the target order. For the latter, only the first occurrence of each target action was considered. For example, on the three-step sequences, if the participant produced all three components in the target order, they would receive credit for three different target actions, and for two correctly ordered pairs of actions (i.e., one point for the Pair 1-2, and one point for the Pair 2-3). If participants produced Actions 1 and 3 in that order, they would receive credit for two different target actions and one correctly ordered pair (i.e., 1 comes before 3). However, if they produced the string of actions 3, 1, 2, 3, they would be credited with three different target actions, but with only one correctly ordered pair: 1-2. They would not be credited with the Pair 2-3, because they already would have been credited with Action 3. This scoring procedure reduces the likelihood of participants receiving credit for production of a sequence by chance or by trial and error.
The two dependent measures, number of target actions and number of action pairs produced, are not independent of one another in that the number of target actions produced affects production of pairs of actions in the target order. Nevertheless, it is possible to earn a high score on the measure of number of different target actions and not earn a high score on the measure of pairs of actions in the target order. In addition, as it is possible to generate target actions in both causal and arbitrary sequences without generating the correct order, participants were credited with an action regardless of the order in which it occurred. For example, in the causal sequence "water transfer," participants were credited with putting their finger on the straw (Step 2) regardless of whether they had put the straw in water (Step 1).

\section{Statistical Analysis}

The number of actions and action pairs correctly recalled was averaged across sequences within each condition. Separate analyses were conducted for the number of target actions (steps) and sequences (action pairs). A significant difference between baseline performance and recall after modeling, in favor of recall, was taken as evidence for memory of the target actions and action pairs. The data were analyzed using an analysis of variance with a between-subjects factor of group (DA, control) and two within-subjects factors of assessment condition (baseline, delay) and sequence structure (causal, arbitrary), with the appropriate tests of normality and homogeneity of variance. All significant interactions were followed up using separate $t$ tests with Bonferroni correction for multiple comparisons. All findings with a $p$ value of less than .05 are reported, and predicted findings with a $p$ value less than .1 are also described.

\section{APPENDIX}

\begin{tabular}{ll}
\hline Three-step causal & Three-step arbitrary \\
\hline Water transfer & Sound tone \\
Put straw in water & $\begin{array}{l}\text { Pour water from one glass to other } \\
\text { Put finger on straw }\end{array}$ \\
$\begin{array}{l}\text { Hit fork on new glass } \\
\text { empty cup }\end{array}$ & Hit fork on old glass \\
Bernoulli effect & Muller-Lyer demonstration \\
Dryer on & Make long configuration with straws \\
Balloon in air stream & Make short configuration with straws \\
Rotate dryer & Interchange straws
\end{tabular}




\section{APPENDIX. continued}

\begin{tabular}{ll}
\hline Magnet & Drawing a star \\
Turn cup over & Fold paper \\
Clip on magnet & Cut corners \\
Pull string & Draw star \\
Tuning fork & Balance coins \\
Lid on box & One coin at one end of ruler \\
Hit fork on table & One coin at other end \\
Put handle on lid & Balance ruler on block \\
\hline
\end{tabular}

\section{Acknowledgments}

We thank the participants and their families for their continued support of our research, Jean M. Mandler and Laraine McDonough for discussions about the design of the study and for providing us with the details of the event sequences, David Gadian for measuring the hippocampal volumes, and Kling Chong for reviewing all of the scans. Support for A.-L. R. A. was provided by a Medical Research Council PhD Studentship. Additional support was provided by the Wellcome Trust and the National Institute of Mental Health Intramural Research Program/National Institutes of Health/ Department of Health and Human Services.

Reprint requests should be sent to Anna Adlam, Cognition and Brain Sciences Unit, Medical Research Council, 15 Chaucer Road, Cambridge, CB2 2EF, UK, or via e-mail: anna.adlam@ mrc-cbu.cam.ac.uk.

\section{Notes}

1. The $p$ value with Bonferroni correction is .01 (.05/5).

2 . The difference between the groups would reach conventional levels of significance uncorrected (i.e., $p=.028$ ). Therefore, in order to rule out any affect of baseline differences on delayed recall performance, an analysis of covariance was carried out to covary baseline performance. This analysis still showed a main effect of group at delayed recall $[F(1,20)=24.4, p<.05]$, indicating that any potential differences at baseline did not account for the effect at delay. 3. The $p$ value with Bonferroni correction is .025 (.05/2).

\section{REFERENCES}

Adlam, A.-L. R., Gadian, D. G., Vargha-Khadem, F., \& de Haan, M. (2004). Deferred imitation of action sequences in developmental amnesia. Paper presented at the Cognitive Neuroscience Society, San Francisco.

Barr, R., \& Hayne, H. (1996). The effect of event structure on imitation in infancy: Practice makes perfect? Infant Behavior and Development, 19, 253-257.

Bauer, P. J. (1992). Holding it all together: How enabling relations facilitate young children's event recall. Cognitive Development, 7, 1-28.

Bauer, P. J. (1996). What do infants recall of their lives? Memory for specific events by one- to two-year-olds. American Psychologist, 51, 29-41.
Bauer, P. J. (1997). Development of memory in early childhood. In N. Cowan (Ed.), The development of memory in childhood (pp. 83-113). Hove, East Sussex, UK: Psychology Press.

Bauer, P. J., \& Hertsgaard, L. A. (1993). Increasing steps in recall of events: Factors facilitating immediate and long-term memory in 13.5- and 16.5-month-old children. Child Development, 64, 1204-1223.

Bauer, P. J., Hertsgaard, L. A., \& Wewerka, S. S. (1995). Effects of experience and reminding on long-term recall in infancy: Remembering not to forget. Journal of Experimental Child Psychology, 59, 260-298.

Bauer, P. J., \& Mandler, J. M. (1992). Putting the horse before the cart: The use of temporal order in recall of events by one-year-old children. Developmental Psychology, 28, 441-452.

Channon, S., \& Daum, I. (2000). The effect of semantic categorisation on recall memory in amnesia. Behavioral Neurology, 12, 107-117.

Channon, S., Daum, I., \& Polkey, C. E. (1989). The effect of categorization on verbal memory after temporal lobectomy. Neuropsychologia, 27, 777-785.

Colvin, M. K., Dunbar, K., \& Grafman, J. (2001). The effects of frontal lobe lesions on goal achievement in the water jug task. Journal of Cognitive Neuroscience, 13, 1129-1147.

Gadian, D. G., Aicardi, J., Watkins, K. E., Porter, D. A., Mishkin, M., \& Vargha-Khadem, F. (2000). Developmental amnesia associated with early hypoxic-ischaemic injury. Brain, 123, 499-507.

Goel, V., \& Grafman, J. (1995). Are the frontal lobes implicated in "planning" functions? Interpreting data from the Tower of Hanoi. Neuropsychologia, 33, 623-642.

Mandler, J. M. (1990). Recall of events by preverbal children. Annals of the New York Academy of Sciences, 608, 485-503.

Mandler, J. M., \& McDonough, L. (1995). Long-term recall of event sequences in infancy. Journal of Experimental Child Psychology, 59, 457-474.

McDonough, L., Mandler, J. M., McKee, R. D., \& Squire, L. R. (1995). The deferred imitation task as a nonverbal measure of declarative memory. Proceedings of the National Academy of Sciences, U.S.A., 92, 7580-7584.

Meltzoff, A. N. (1988). Imitation of televised models by infants. Child Development, 59, 1221-1229.

Nelson, C. A. (2000). Neural plasticity and human development: The role of early experience in sculpting memory systems. Developmental Science, 3, 115-136.

Rey, A. (1964). L'examen clinique en psychologie. Paris: Presses Universitaire de France.

Rust, J., Golombok, S., \& Trickey, G. (1993). Wechsler objective reading dimensions. Sidcup, Kent: The Psychological Corporation.

Strange, B. A., Fletcher, P. C., Henson, R. N., Friston, K. J., \& Dolan, R. J. (1999). Segregating the functions of human hippocampus. Proceedings of the National Academy of Sciences, U.S.A., 96, 4034-4039.

Talley, J. L. (1993). Children's auditory verbal learning test. Odessa, FL: Psychological Assessment Research.

Tulving, E. (1972). Episodic and semantic memory. In E. Tulving \& W. Donaldson (Eds.), Organization of memory (pp. 381-403). New York: Plenum.

Vargha-Khadem, F., Gadian, D. G., \& Mishkin, M. (2001). Dissociations in cognitive memory: the syndrome of developmental amnesia. Philosophical Transactions of the Royal Society of London. Series B, 356, 1435-1440.

Vargha-Khadem, F., Gadian, D. G., Watkins, K. E., Connelly, A., Van Paesschen, W., \& Mishkin, M. (1997). Differential effects of early hippocampal pathology on episodic and semantic memory. Science, 277, 376-380. 
Vargha-Khadem, F., Isaacs, E., van der Werf, S., Robb, S., \& Wilson, J. (1992). Development of intelligence and memory in children with hemiplegic cerebral palsy. The deleterious consequences of early seizures. Brain, 115, 315-329.

Vargha-Khadem, F., Salmond, C. H., Watkins, K. E., Friston, K. J., Gadian, D. G., \& Mishkin, M. (2003). Developmental amnesia: effect of age at injury. Proceedings of the National Academy of Sciences, U.S.A., 100, 10055-10060.

Wechsler, D. (1992). Manual for the Wechsler Intelligence
Scale for Children (3rd ed., UK ed.). London: The Psychological Corporation.

Wechsler, D. (1997). Manual for the Wechsler Adult Intelligence Scale-III. London: The Psychological Corporation.

Werker, J. (1990). Commentary on "Recall of events by preverbal children" by Mandler, J. M. (1990). Annals of the New York Academy of Sciences, 608, 485-516.

Wilson, B., Cockburn, J., \& Baddeley, A. (1985). Rivermead Behavioral Memory Test. Reading, UK: Thames Valley Test Company. 